\title{
LOS CAMPOS GEOTÉRMICOS DE CENTROAMÉRICA: INFLUENCIA DEL PROCESO DE SUBDUCCIÓN SOBRE SU COMPOSICIÓN VOLÁTIL
}

\author{
Glen Snyder ${ }^{1,2 *}$, Robert Poreda ${ }^{1}$, Udo Fehn $^{1} \&$ Andrew Hunt ${ }^{1,3}$ \\ ${ }^{1}$ Dept. Earth \& Environmental Sciences , University of Rochester, \\ Rochester, NY, 14627, EE.UU. \\ 2 Dirección actual: Rice University, Earth Science-MS126, P.O. Box 1892, \\ Houston, TX 77251-1892, EE.UU. \\ ${ }^{3}$ Dirección actual: U.S. Geological Survey, Bldg. 21, MS963, \\ Denver Federal Center, Denver, CO 80225, EE.UU. \\ * Autor para contacto: gsnyder@ rice.edu
}

(Recibido 12/11/02; aceptado 27/01/03)

\begin{abstract}
Gases from geothermal production wells in Costa Rica, Nicaragua, and El Salvador were collected and analyzed for major and noble gas composition in order to determine the sources of volatiles in active Central American volcanic systems. Ratios of ${ }^{3} \mathrm{He} /{ }^{4} \mathrm{He}$ indicate that the upper mantle is the principal source of helium, with minor amounts derived from the crust in the Berlín field of El Salvador. The Miravalles field of Costa Rica is characterized by high $\mathrm{CO}_{2} /{ }^{\beta} \mathrm{He}$ ratios which are likely a product of increased devolatilization in the southwestern portion of the Cocos plate. The Momotombo field of Nicaragua shows high nitrogen and methane concentrations, both of which are likely the product of local contributions within the Nicaraguan Depression. The Central American arc is, in general, characterized by high $\mathrm{N}_{2}{ }^{3} \mathrm{He}$ ratios and high ${ }^{3} \mathrm{He} /{ }^{4} \mathrm{He}$ ratios, a combination which is unusual for island arc systems. The results demonstrate that understanding the gas systematics in geothermal systems, both during initiation and during commercial production, is essential in the planning and maintenance of this important resource.
\end{abstract}

Keywords: Central America, geothermal fields, volatiles, noble gases, upper mantle, geothermal production.

RESUMEN: Se recolectaron gases de pozos de producción geotérmica en Costa Rica, Nicaragua y El Salvador, y se analizó su contenido de gases mayores y gases nobles, con el fin de determinar las fuentes de los volátiles dentro de los sistemas volcánicos activos de América Central. Las razones de ${ }^{3} \mathrm{He} /{ }^{4} \mathrm{He}$ indican que el manto superior es la fuente principal de helio, con cantidades menores provenientes de la corteza en el campo de Berlín en El Salvador. El campo de Miravalles en Costa Rica se caracteriza por altas relaciones de $\mathrm{CO}_{2} /{ }^{3} \mathrm{He}$, las cuales probablemente son producto del aumento de devolatilización en la parte suroeste de la placa del Coco. El campo de Momotombo en Nicaragua demuestra un alto contenido de nitrógeno y metano, ambos probablemente el producto de contribuciones locales dentro de la depresión de Nicaragua. El arco volcánico de América Central se caracteriza, por lo general, por altas razones de $\mathrm{N}_{2} /{ }^{3} \mathrm{He}$ y ${ }^{3} \mathrm{He} /{ }^{4} \mathrm{He}$, una combinación poco usual para los sistemas de arco de islas. Los resultados demuestran que un buen entendimiento de la sistemática de los gases en los sistemas geotérmicos, durante su inicio y su producción comercial, es esencial para la planificación y mantenimiento de este importante recurso.

Palabras clave: América Central, campos geotérmicos, volátiles, gases nobles, manto superior, producción geotérmica. 


\section{INTRODUCCIÓN}

El transporte de los elementos volátiles a través de las zonas de subducción contribuye a los procesos responsables de la formación de los volcanes del tipo arco de islas. Una porción importante de los gases magmáticos en dichos sistemas (por ejemplo $\mathrm{CO}_{2}, \mathrm{H}_{2} \mathrm{O}, \mathrm{N}_{2}$ y los gases nobles) proviene de los sedimentos y basaltos alterados de la corteza oceánica, en el caso de América Central de la placa del Coco (Fig. 1). Durante su subducción debajo de la placa del Caribe, la placa del Coco experimenta un aumento de temperatura, lo que provoca su deshidratación y devolatilización. Los fluidos derivados de tal acción son de interés porque inducen la fusión parcial de la sección del manto superior que existe entre la porción de la placa del Coco que desciende y la sobreyacente placa del Caribe. Al ascender el magma que se origina debajo de los volcanes de América Central, la disminución de presión permite la separación de las fases de magma líquido y gas, provocando lo que a veces son erupciones violentas. Así, las fumarolas, las pailas burbujeantes de lodo y aguas termales que se observan en América

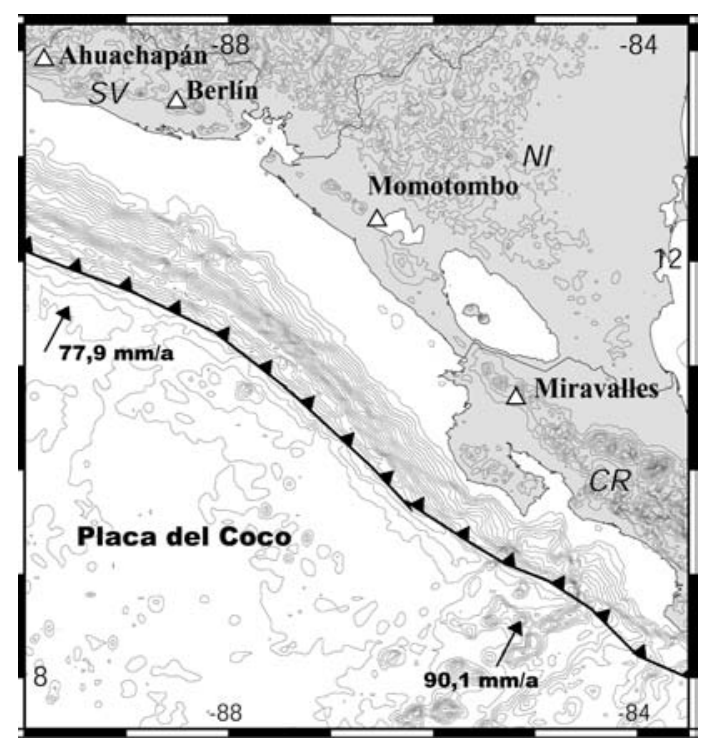

Fig. 1: Mapa regional de América Central, con la ubicación de los principales campos geotérmicos en relación con la Fosa Mesoamericana y la placa del Coco.
Central derivan sus componentes gaseosos, en gran parte, de los materiales volátiles relacionadas con la subducción de la placa del Coco y del manto superior. En cambio, el vapor de agua que viene asociado con tales manifestaciones termales tiene un origen primariamente relacionado con la interacción entre las aguas subterráneas, los gases magmáticos y las rocas calentadas en el acuífero.

La composición de los elementos volátiles debajo de la cadena volcánica depende de la composición de los sedimentos marinos (Patino et al., 2000; Fischer et al., 2002), tanto como el estado térmico y geométrico de la placa del Coco (Leeman \& Carr, 1995). Ciertos elementos, como el carbón, se liberan en menores cantidades de los carbonatos marinos llevados por la subducción a una profundidad de aproximadamente $100 \mathrm{~km}$ debajo de la zona volcánica, mientras que otros elementos, como el nitrógeno y los gases nobles, son enteramente liberados en las zonas antearco y debajo de los arcos de islas. Tales diferencias han tenido un impacto en el inventario de los gases atmosféricos a nivel global a través de millones de años. Hoy día, el manto superior ha perdido aproximadamente el 99\% de su argón-36 y 97\% de su helio-3 primordial, ambos por la desgasificación que ocurre a lo largo de las serranías oceánicas submarinas. Sin embargo, el manto superior ha perdido solamente el $28 \%$ de su total de $\mathrm{CO}_{2}$, debido a que gran parte del $\mathrm{CO}_{2}$ es continuamente reincorporado por la subducción a profundidades mayores de los $100 \mathrm{~km}$ en el manto. De manera similar, la pérdida de nitrógeno primordial del manto, es alrededor del $88 \%$, lo cual sugiere que el nitrógeno también se recicla en el manto por la subducción (Zhang \& Zindler, 1993; Kerrick \& Connolly, 2001). Varias investigaciones han incorporado la sistemática de los gases nobles y los isótopos estables para determinar el porcentaje de contribución de $\mathrm{CO}_{2}$ y $\mathrm{N}_{2}$ del manto superior y de los sedimentos que han pasado por la subducción (por ejemplo: Sano \& Williams, 1996; Sano et al., 2001); y éstos han sido utilizados para extrapolar la contribución volcánica global de cada gas (p.ej.: Hilton et al., 2002).

En el caso de América Central, donde la explotación geotérmica suple una porción significativa de la demanda eléctrica, existe un importante 
interés en las interacciones de los gases magmáticos con las aguas subterráneas. Tal interacción tiene impacto en el potencial de producción de cada campo. Por ejemplo, la presencia del $\mathrm{CO}_{2}$ y su desgasificación en los campos, provoca la precipitación de calcita dentro de las perforaciones geotérmicas. El equilibrio completo o parcial entre los componentes magmáticos disueltos en las aguas subterráneas con las rocas adyacentes es, frecuentemente, una función de la temperatura, que puede ser aplicada a la geotermometría. La comparación de varios geotermómetros con las temperaturas máximas de los pozos puede dar una indicación del tiempo de residencia de las aguas en el lugar donde se sitúan actualmente.

Presentamos una parte de las investigaciones que llevamos a cabo entre 1997 y 1999 en América Central gracias al apoyo logístico brindado por el Instituto Costarricense de Electricidad (ICE), el Instituto Nacional de Estudios Territoriales (INETER, Nicaragua), la Empresa Nicaragüense de Electricidad (ENEL) y Geotérmica Salvadoreña (GESAL). Durante este periodo, recolectamos 37 muestras de gas de pozos de producción y 4 muestras de fumarolas y aguas termales. Además, tomamos 48 muestras de agua separada de pozos de producción, 16 de fumarolas y aguas termales, 5 de lagunas cratéricas y 8 de ríos y lagunas. Los pozos de producción tienen la ventaja de permitir el muestreo de aguas y gases a una profundidad entre $500 \mathrm{~m}$ y $2000 \mathrm{~m}$, a temperaturas que generalmente varían entre $200^{\circ} \mathrm{C}$ y $350^{\circ} \mathrm{C}$, cuya contribución de aguas meteóricas recientes es mínima. Para una descripción más detallada sobre los resultados de la investigación, pueden referirse a Snyder et al. (2001, 2003) y Snyder \& Fehn (2002). Tales publicaciones dan más detalle sobre los métodos utilizados y las implicaciones en cuanto a la producción global de gases volcánicos y su impacto en los ciclos globales de nitrógeno y el carbón. En cambio, el enfoque de este estudio es en las variaciones de composición que existen dentro de cada campo geotérmico y su relación con la temperatura dentro de cada sitio.

\section{LOS CAMPOS DE PRODUCCIÓN GEOTÉRMICA EN CENTROAMÉRICA}

\section{El Salvador}

El campo geotérmico de Ahuachapán se ubica en la región occidental de El Salvador, cerca de la frontera con Guatemala (Fig. 1), siendo el campo geotérmico de producción eléctrica más antiguo en Centroamérica. La producción comenzó en el año 1975, con dos estaciones generadoras bajo el manejo de la Comisión Ejecutiva del Río Lempa (CEL). El campo se sitúa dentro del Graben Central, el cual se ubica al norte de la cordillera volcánica. Inicialmente, las salmueras fueron reinyectadas, hasta que se construyó un canal de drenaje hasta el océano en el año 1982 (Stefánsson, 1997). Las lavas y depósitos piroclásticos llegan a tener más de $500 \mathrm{~m}$ de espesor, relacionados con la erupción pleistocena del volcán Cerro Laguna Verde. Las lavas profundas tienen baja permeabilidad, así que la circulación de los fluidos es dominantemente por flujo a lo largo de las fracturas. La ubicación de los pozos se relaciona con las fallas este-oeste. Las temperaturas se encuentran entre $230^{\circ} \mathrm{C}$ y $240^{\circ} \mathrm{C}$ y la profundidad varía entre los 400 y 600 m. La producción total supera los $100 \mathrm{MW}$ (Vimmerstedt, 1998).

Las investigaciones del campo geotérmico de Berlín comenzaron en el año 1976 (Cuéllar, 1981) y actualmente existen cuatro pozos con una producción total de 26,7 MW (D’Amore \& Mejía, 1999; Montalvo \& Axelsson, 2000). Los pozos se ubican dentro de la caldera de Berlín y lo que queda de la última actividad del volcán Berlín desde hace $100 \mathrm{ka}$. La caldera cubre un área aproximada de 6-10 km² (Matus et al., 1999) y, al igual que Ahuachapán, se sitúa dentro del Graben Central. El reservorio geotérmico se sitúa dentro de lavas andesíticas, lavas andesítico-basálticas y tobitas terciarias (Mejía et al., 1997). Las temperaturas del reservorio varían entre $240^{\circ} \mathrm{C}$ y $310^{\circ} \mathrm{C}$ y la profundidad de los pozos es entre 1500 y $2400 \mathrm{~m}$ (D’Amore \& Mejía, 1999).

En años recientes, la tendencia hacia la privatización del sector energético en América 
Central ha resultado en importantes cambios en cuanto a las investigaciones, el desarrollo, y la administración de los campos geotérmicos (Bundschuh et al., 2002). El grupo de generación geotérmica salvadoreña fue separada del CEL y existe hoy día como una empresa subsidiaria denominada Geotérmica Salvadoreña (GESAL).

\section{Nicaragua}

Las investigaciones y el desarrollo del campo geotérmico de Momotombo comenzaron en el costado suroeste del volcán en el año 1974, mientras que la producción de electricidad se inició en 1981 (Adams et al., 1981; Moore et al., 1981). Se ha cumplido la perforación de 40 pozos dentro de un área de $5 \mathrm{~km}^{2}$ durante los últimos 25 años, con profundidades entre 400 y $2480 \mathrm{~m}$. El volcán Momotombo queda en el sur de la cordillera volcánica de los Marrabios, dentro de la depresión de Nicaragua. Las rocas volcánicas recuperadas de la perforación de los pozos incluyen andesitas basálticas, tobitas y brechas del Terciario hasta el Cuaternario con delgados niveles de calizas que incluyen corales y percebes marinos a los $2200 \mathrm{~m}$ (Moore et al., 1981). La producción total es de 30 MW (Vimmerstedt, 1998). Concesiones del campo geotérmico de Momotombo, junto con la operación de la planta generadora, fueron privatizados en el año 2000 bajo la administración del Instituto Nicaragüense de Energía (INE). La ENEL se dividió en varías empresas dedicadas a la generación, la transmisión y la distribución de electricidad (Bundschuh et al., 2002).

\section{Costa Rica}

Las investigaciones geotérmicas en detalle comenzaron en la provincia de Guanacaste durante el año 1975, con los primeros pozos de prueba perforados en Miravalles en 1979 (Giggenbach \& Corrales, 1992). La producción de electricidad comenzó a principios de los años 1990. El campo geotérmico se ubica dentro de la caldera de Guayabo, que es una depresión de más de $8 \mathrm{~km}$ de diámetro de edad pleistocena, al costado occidental del volcán Miravalles. La actividad más reciente del volcán es de unos 7 ka. Los pozos varían desde 1162 hasta los $2270 \mathrm{~m}$ de profundidad y tienen temperaturas máximas de $240^{\circ} \mathrm{C}$. Los pozos más profundos perforan una secuencia de depósitos volcaniclásticos y laháricos, tobitas y lavas dacíticas y andesíticas (Bargar \& Fournier, 1988; Grigsby et al., 1989). La expansión reciente del campo ha aumentado la producción hasta $110 \mathrm{MW}$, con concesiones otorgadas a una compañía privada para una planta adicional de 27,5 MW. Para el futuro, se proyecta el desarrollo de campos de producción en los volcanes adyacentes (Mainieri, 2000).

\section{EL HELIO Y EL ARGÓN EN LOS SISTEMAS GEOTÉRMICOS}

La composición isotópica del helio sirve como indicador de la proporción de gas magmático versus la cantidad derivada de la corteza o de los sedimentos, debido a que el ${ }^{3} \mathrm{He}$ es primariamente de origen primordial, que aún existe en el manto superior, mientras que el ${ }^{4} \mathrm{He}$ es radiogénico, producido in situ dentro de la corteza. Frecuentemente, se normalizan las tasas $\mathrm{de}{ }^{3} \mathrm{He} /{ }^{4} \mathrm{He}$ con la del aire $\left({ }^{3} \mathrm{He} /{ }^{4} \mathrm{He}=1,40 \times 10^{-6}\right)$. Además, se puedan corregir las tasas de ${ }^{3} \mathrm{He} /{ }^{4} \mathrm{He}$ por la contaminación del aire, utilizando el neón como indicador del grado de contaminación atmosférica que existe debido a la incorporación de gases al momento del muestreo. Valores típicos para ${ }^{3} \mathrm{He} /{ }^{4} \mathrm{He}$ del manto superior y la corteza oceánica son de $\sim 8 \mathrm{R}_{\text {aire }}$ mientras que el helio en granitos $\mathrm{u}$ otras rocas ricas en uranio, suele ser de $\sim 0,01 \mathrm{a} \sim 0,1 \mathrm{R}_{\text {aire }}$. En Centroamérica, por lo general, son poco variables y las relaciones oscilan entre 6,25 y 7,25 $\mathrm{R}_{\text {aire }}$ en Miravalles, Momotombo y Ahuachapán (Cuadro 1). El único lugar donde se nota una contribución apreciable del ${ }^{4} \mathrm{He}$, es en Berlín, donde las tasas son de $5,6 \mathrm{R}_{\text {aire }}$ (Snyder et al., 2001). Esto contrasta con los gases de las aguas termales detrás del frente volcánico en Honduras, donde la influencia de la corteza, aparentemente del bloque Chortis, resulta en tasas de helio de 0,55 a 3,08 $\mathrm{R}_{\text {aire }}$ (Kennedy et al., 1991; Goff et al., 1987). 
Cuadro 1

Valores medios* de la composición del gas para los campos geotérmicos de América Central

\begin{tabular}{lllllll}
\hline & $\begin{array}{c}{ }^{4} \mathrm{He} /{ }^{3} \mathrm{He} \\
\left(\mathrm{R}_{\text {aire }}\right)\end{array}$ & $\begin{array}{c}{ }^{40} \mathrm{Ar} /{ }^{36} \mathrm{Ar} \\
(\%)\end{array}$ & $\begin{array}{c}\delta^{13} \mathrm{C}_{\mathrm{co} 2} \\
\left(10^{9}\right)\end{array}$ & $\begin{array}{c}\mathrm{CO}_{2} /{ }^{\beta} \mathrm{He} \\
\left(10^{6}\right)\end{array}$ & $\mathrm{CH}_{4}{ }^{\beta} \mathrm{He}$ & $\mathrm{N}_{2} / \mathrm{Ar}$ \\
\hline Miravalles, CR & $6,6 \pm 0,1$ & $300 \pm 4$ & $-1,09 \pm 0,02$ & $55,9 \pm 5,3$ & $16,2 \pm 4,9$ & $83 \pm 11$ \\
Momotombo, NI & $6,8 \pm 0,0$ & $300 \pm 3$ & $-1,22 \pm 0,09$ & $24,7 \pm 1,3$ & $108,0 \pm 6,5$ & $314 \pm 82$ \\
Berlín, ES & $5,6 \pm 0,1$ & $299 \pm 2$ & $\mathrm{ND}$ & $23,6 \pm 2,3$ & $9,3 \pm 2,7$ & $79 \pm 9$ \\
Ahuachapán, ES & $7,0 \pm 0,2$ & $296 \pm 1$ & $-2,30 \pm 0,13$ & $17,7 \pm 4,8$ & $6,6 \pm 1,7$ & $64 \pm 21$ \\
Arco de islas "típico" & 7,0 & 300 & $<0,0$ & 20 & --- & 200 \\
Manto superior & 8,0 & 28000 & $-6,5$ & 1,5 & --- & 94 \\
Agua saturada de aire & 1,0 & 295 & --- & & --- & 38 \\
\hline
\end{tabular}

* Para una lista completa de los datos, referirse a Snyder et al. (2001, 2003) y Snyder \& Fehn (2002). Valores del arco de islas típico, el manto superior, y el agua saturada por aire tomadas de: Varekamp et al. (1992), Farley \& Poreda (1993), Zhang \& Zindler (1993), Giggenbach \& Poreda (1993), Sano \& Marty (1995), Sano \& Williams (1996), Sano et al.(2001)

Por lo general, existe una leve disminución entre las tasas de ${ }^{3} \mathrm{He} /{ }^{4} \mathrm{He}$ en los pozos que presentan una temperatura más baja, debido a que la contribución de helio magmático es menor. Esta tendencia se ve entre los pozos de Ahuachapán (Fig. 2) y Momotombo (Fig. 3). Presentamos las tasas de ${ }^{3} \mathrm{He} /{ }^{4} \mathrm{He}$ comparado con los valores de entalpía derivados de geotemperaturas, los cuales son calculados a partir de las concentraciones de $\mathrm{Na}, \mathrm{K}, \mathrm{y} \mathrm{Ca}$ en las aguas (Fournier \& Truesdell, 1973). El caso de Miravalles es menos claro y el campo parece estar dividido en una región al este con la relación ${ }^{3} \mathrm{He} /{ }^{4} \mathrm{He}$ más alta, y una al oeste, que es más baja (Fig. 4).

Al igual que el helio, el argón tiene una composición isotópica que se puede utilizar para distinguir entre diferentes componentes que contribuyen a la composición de los gases. En este caso, el argón del manto es enriquecido en ${ }^{40} \mathrm{Ar}$, que se obtiene de la descomposición del ${ }^{40} \mathrm{~K}$ relativo al ${ }^{36} \mathrm{Ar}$ primordial. La relación de ${ }^{40} \mathrm{Ar} /{ }^{36} \mathrm{Ar}$ en la corteza oceánica y el manto superior es alrededor de 28 000. En cambio, la tasa atmosférica es de 295, y las aguas superficiales conservan esta tasa, ya que contienen argón atmosférico disuelto (Farley \& Poreda, 1993; Zhang \& Zindler, 1993). Los pozos geotérmicos de Centroamérica tienen tasas de ${ }^{40} \mathrm{Ar} /{ }^{36} \mathrm{Ar}$ que varían entre 283 y 308.

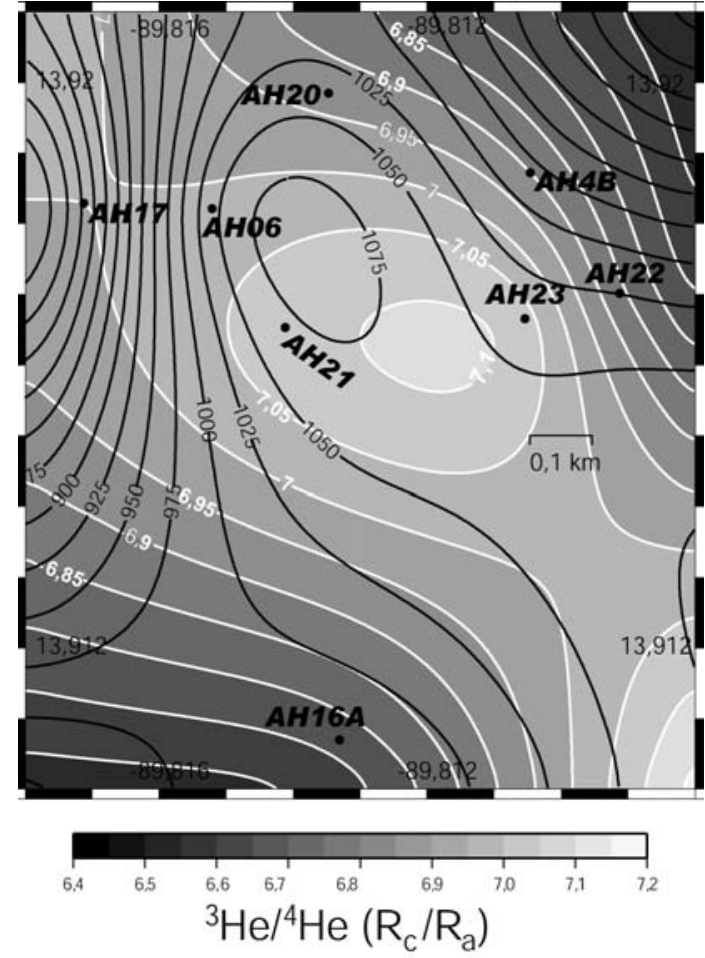

Fig. 2: Variaciones de ${ }^{3} \mathrm{He} /{ }^{4} \mathrm{He}$ (corregido por la contaminación del aire) dentro del campo geotérmico de Ahuachapán, El Salvador. Las líneas sólidas representan la entalpía, en $\mathrm{kJ} / \mathrm{kg}$, determinado con las temperaturas de $\mathrm{Na}-\mathrm{K}-\mathrm{Ca}$. 


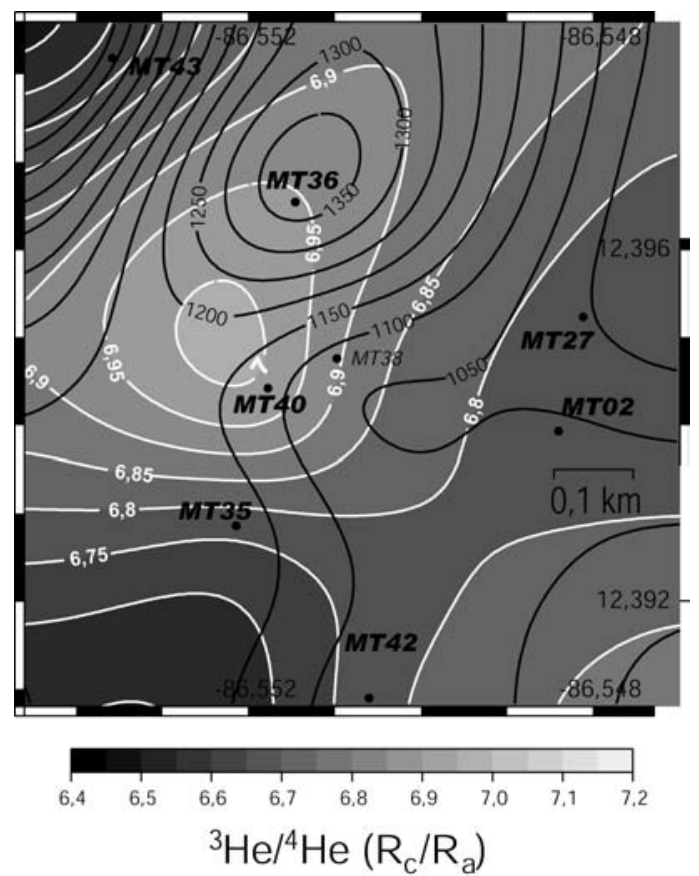

Fig. 3: Variaciones de ${ }^{3} \mathrm{He} /{ }^{4} \mathrm{He}$ en el campo geotérmico de Momotombo, Nicaragua. Los pozos con mayor ${ }^{3} \mathrm{He} /{ }^{4} \mathrm{He}$ se aproximan a los de mayor entalpía, aunque no existe relación directa

Las fumarolas y aguas termales tienen una relación ${ }^{40} \mathrm{Ar} /{ }^{36} \mathrm{Ar}$ parecida, lo cual indica que la fuente predominante de Ar en estos sistemas es de origen meteórico, ya disuelto en las aguas subterráneas.

\section{$\mathrm{EL} \mathrm{CO}_{2} \mathrm{Y} \mathrm{CH}_{4} \mathrm{EN}$ SISTEMAS GEOTÉRMICOS}

Después del vapor de agua, el $\mathrm{CO}_{2}$ es el componente más importante entre las fumarolas y los pozos geotérmicos. El hecho de que el $\mathrm{CO}_{2}$ predomina sobre los otros gases, junto a la semejanza de su composición isotópica del carbón $\left(\delta^{13} \mathrm{C}\right)$ con las calizas marinas, apoya el concepto de que los sedimentos pasan por la subducción para emerger como volátiles volcánicos (Sano \& Marty, 1995; Varekamp et al., 1992; Sano \& Williams, 1996; Snyder et al., 2001; Hilton et al., 2002). Es muy frecuente presentar la información del bióxido de carbono normalizado con helio-3, o sea $\mathrm{CO}_{2} /{ }^{3} \mathrm{He}$,
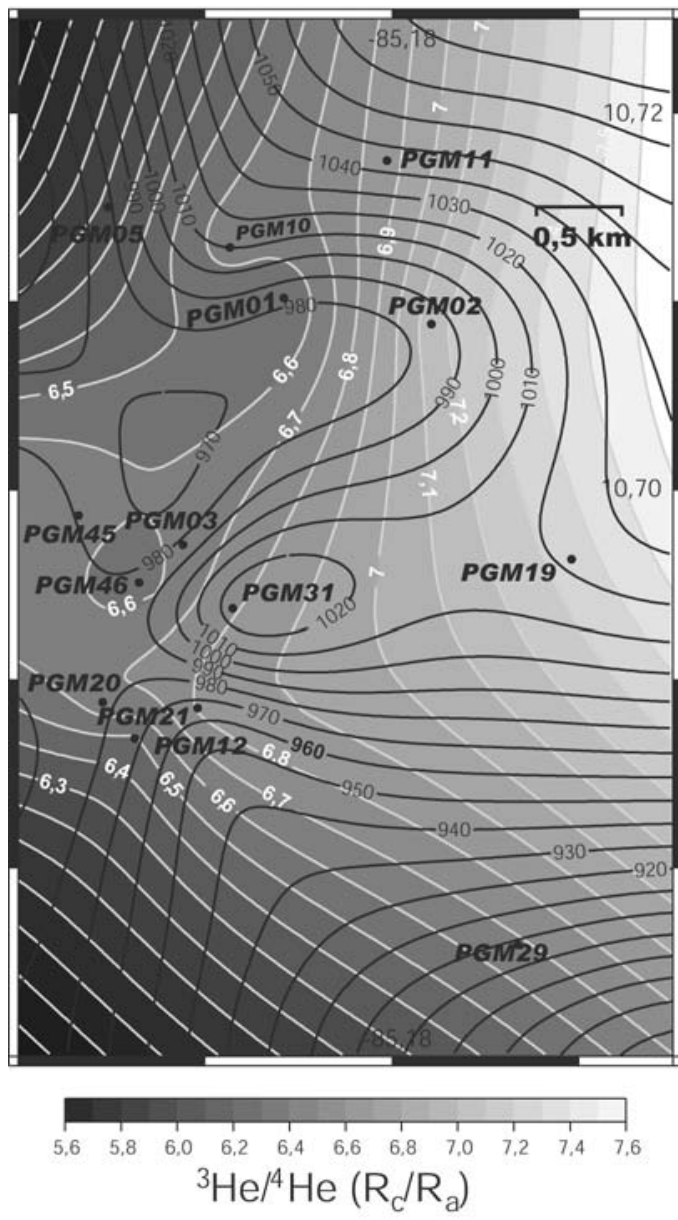

Fig. 4: Variaciones de ${ }^{3} \mathrm{He} /{ }^{4} \mathrm{He}$ dentro del campo geotérmico de Miravalles, Costa Rica. Los patrones son complicados, ya que los pozos de alta entalpía en la parte central de la zona de estudio tienen tasas de ${ }^{3} \mathrm{He} /{ }^{4} \mathrm{He}$ más bajas.

ya que la solubilidad de ambos gases en el magma es parecida y se presume que el proceso de fraccionamiento de ambos durante el proceso de devolatilización de un cuerpo magmático es mínimo.

En casi todos los volcanes en los márgenes del Pacífico existe una mezcla binaria predominante entre carbono del manto superior $\left(\mathrm{CO}_{2}{ }^{\beta} \mathrm{He}=1,50 \times 10^{9} ; \delta^{13} \mathrm{C}=-6,5 \%\right.$ ) y carbono de las calizas marinas $\left(\mathrm{CO}_{2}{ }^{\beta} \mathrm{He}=1 \times 10^{13} ; \delta^{13} \mathrm{C}=0 \%\right.$, Sano \& Williams, 1996). La causa de las divergencias de la mezcla directa de los dos $\left(\mathrm{CO}_{2} /{ }^{\beta} \mathrm{He}\right.$ alta, con $\delta^{13} \mathrm{C}<0 \%$ ) ha sido explicada como el resultado de la influencia de material orgánico que tiene 


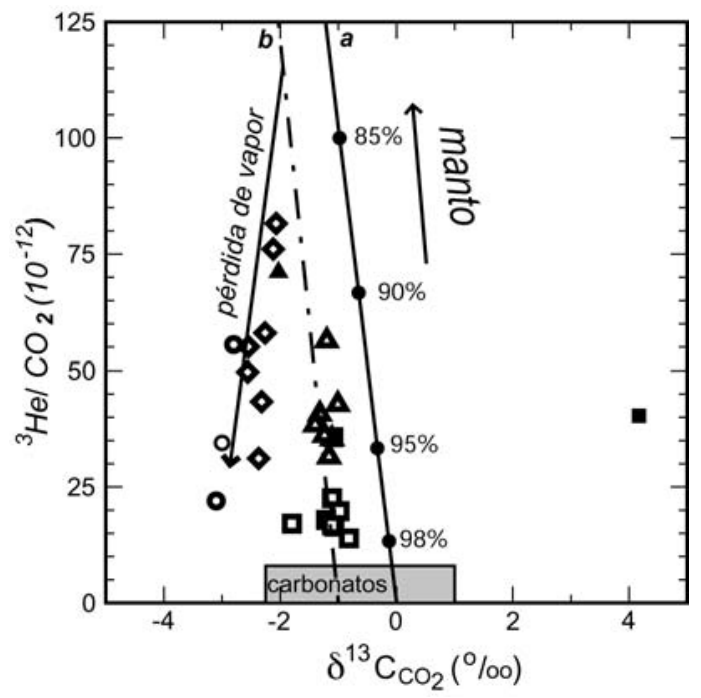

Fig. 5: Las composiciones de ${ }^{3} \mathrm{He} / \mathrm{CO}_{2}$ y $\delta^{13} \mathrm{C}$ están controladas por: 1) la mezcla de $\mathrm{CO}_{2}$ de los carbonatos de la placa del Coco que han pasado por la subducción con $\mathrm{CO}_{2}$ del manto superior y 2) la mezcla de los dos da origen al componente magmático. El otro factor es la pérdida preferencial de helio y la precipitación de carbonato dentro de los acuíferos, ya disuelto el componente magmático en las aguas subterráneas. Cuadrados $=$ Miravalles, triángulos $=$ Momotombo, círculos $=$ Berlín, diamantes = Ahuachapán, triángulo sólido= aguas termales, cuadrado sólido = fumarola de baja temperatura Volcán Poás.

una relación ${ }^{13} \mathrm{C} /{ }^{12} \mathrm{C}$ menor a la de los carbonatos (Sano \& Marty, 1995). Nuestras investigaciones (Snyder et al., 2001), sugieren que las diferencias de $\delta^{13} \mathrm{C}$ y $\mathrm{CO}_{2} /{ }^{\beta} \mathrm{He}$ son hasta cierto punto influenciadas por interacciones dentro de las aguas subterráneas. Presentamos la misma línea de mezcla, pero con el recíproco de la composición $\left({ }^{3} \mathrm{He} / \mathrm{CO}_{2}\right.$ para el manto superior es igual a $6,7 \times 10^{-10}$, mientras que ${ }^{3} \mathrm{He} / \mathrm{CO}_{2}$ para los carbonatos es apenas de $1 \times 10^{-13}$ ). Esto nos permite convertir la mezcla en una línea recta y enfocarnos más en los cambios que ocurren cerca del carbonato (Fig. 5). Esta línea indica que el carbonato es de una composición isotópica un poco más liviana $\left(\delta^{13} \mathrm{C}=-1\right)$. También indica que la influencia de los carbonatos es mayor en Costa Rica que en Nicaragua y El Salvador. Dicha observación confirma la idea que la placa del Caribe es más joven en el sur de América Central, lo cual puede ocasionar una devolatilización mayor de los carbonatos, a una temperatura mayor. En cambio, muchos de los pozos geotérmicos en El Salvador salen de la línea de mezcla. Esto es debido a que la solubilidad del helio en las aguas subterráneas es 50 veces menor a la del $\mathrm{CO}_{2}$, y la pérdida del vapor de tales sistemas mueve la tasa de ${ }^{3} \mathrm{He} / \mathrm{CO}_{2}$ a valores de saturación de $\sim 0,3 \times 10^{-12}$ (Giggenbach \& Poreda, 1993). A la vez, la precipitación de carbonatos que acompaña a la pérdida de $\mathrm{CO}_{2}$ resulta en una leve baja en los valores de $\delta^{13} \mathrm{C}$ del $\mathrm{CO}_{2}$ residual. Entonces, la distribución de $\mathrm{CO}_{2}{ }^{\beta} \mathrm{He}$ puede ser utilizada como indicador de las regiones donde menos se han perdido sus gases (Fig. 6).

Cabe mencionar que, mientras los campos geotérmicos de El Salvador presentan evidencia de pérdida preferencial del helio, no existen suficientes datos para determinar si éste es producto de la explotación de los pozos o si es un proceso que precede al desarrollo comercial del campo geotérmico. Cambios en la concentración de cloruro, en los valores de $\delta^{18} \mathrm{O}$, y en la entalpía durante varios años de producción, han sido reportados en el campo de Berlín, El Salvador (Montalvo \& Axelsson,

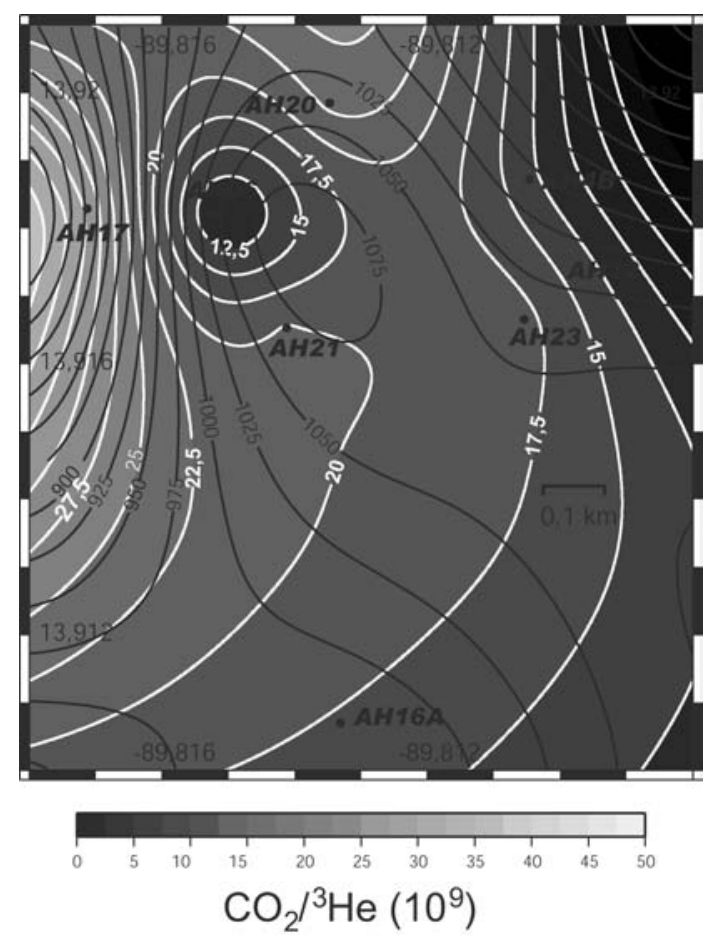

Fig. 6: Valores altos de $\mathrm{CO}_{2}{ }^{\beta} \mathrm{He}$ en Ahuachapán, corresponden con los pozos de baja entalpía, ya que son aguas que han perdido parte de su componente magmático de helio. 
2000) y en Miravalles, Costa Rica (Yock, 2000). Posibles causas incluyen una baja en la presión y la separación de fases (ebullición) dentro del reservorio durante la extracción de los fluidos, o la influencia de la reinyección de los fluidos separados.

El metano que existe en los sistemas geotérmicos puede formarse en cantidades traza por procesos abióticos, aunque la gran mayoría de metano que se observa en los campos geotérmicos es producto de reacciones termocatalíticas dentro de materia orgánica que reside en sedimentos poco profundos de la corteza (Poreda et al., 1988; Schoell, 1988). Valores típicos de $\delta^{13} \mathrm{C}$ para la producción termogénica varían entre $-46 \%$ y $-32 \%$ y $\mathrm{CH}_{4} /{ }^{3} \mathrm{He}$ varía entre $1 \times 10^{8}$ y 1 x $10^{12}$ (Poreda et al., 1988). Mientras la materia orgánica se agota progresivamente, bajan las tasas de $\mathrm{CH}_{4} /{ }^{3} \mathrm{He}$ y los valores de $\delta^{13} \mathrm{C}$ comienzan a subir hacia valores magmáticos (Giggenbach, 1977). Donde la $\delta^{13} \mathrm{C}$ del $\mathrm{CH}_{4}$ está en equilibrio con la del bióxido de carbono, los valores varían entre $-18 \%$ y $-15 \%$ o (Welhan \& Craig, 1983; Schoell, 1988; Welhan, 1988). El equilibrio isotópico del metano con el $\mathrm{CO}_{2}$ depende de la temperatura (Richet et al., 1977; Horita, 2001). Sin embargo, los sistemas geotérmicos rara vez obtienen este equilibrio, dado que el exceso de metano producido por las reacciones termocatalíticas no tiene tiempo suficiente para equilibrarse (que varía de solamente 12 años a $\operatorname{los} 300^{\circ} \mathrm{C}$, hasta 160000 años a los $100^{\circ} \mathrm{C}$; Giggenbach, 1997). En Momotombo, la adición del metano es significativa, y resulta en un desequilibrio que es mayor al de Miravalles (Fig. 7). Se presume que éste está relacionado con materia orgánica que reside dentro de la depresión de Nicaragua y puede estar relacionado con los hidrocarburos de la Cuenca de Sandino (Darce et al., 2000). Hay una muestra en Miravalles (PGM29) que se parece a las de Momotombo, en cuanto al valor de $\delta^{13} \mathrm{C}$ del metano. Es posible que hay un aporte de sedimentos intracaldéricos, aunque determinamos en un estudio del yodo-129 (Snyder \& Fehn, 2002), que probablemente el componente orgánico de los fluidos es mayor en edad que las erupciones del volcán Miravalles.

\section{NITRÓGENO EN LOS SISTEMAS GEOTÉRMICOS}

Al igual que el argón, el nitrógeno molecular que predomina en los sistemas geotérmicos es en gran parte de origen atmosférico. Debido a esto, es frecuente encontrar valores de $\mathrm{N}_{2} /$ Ar entre 38 (que representa la saturación de agua subterránea de ambos gases) y 86 (que representa la composición atmosférica). Otra importante fuente de nitrógeno, que produce tasas de $\mathrm{N}_{2} /$ Ar mucho mayores que la de la atmósfera, es de la descomposición termogénica de material orgánico en los sedimentos. Puesto que el nitrógeno es frecuentemente el gas más abundante en las manifestaciones termales de los sistemas de tipo arco de islas, varias investigaciones han llegado a la conclusión de que el nitrógeno de sistemas magmáticos puede ser un buen indicador de la subducción de materia orgánica (Sano et al., 2001) y que las variaciones en la composición a lo largo

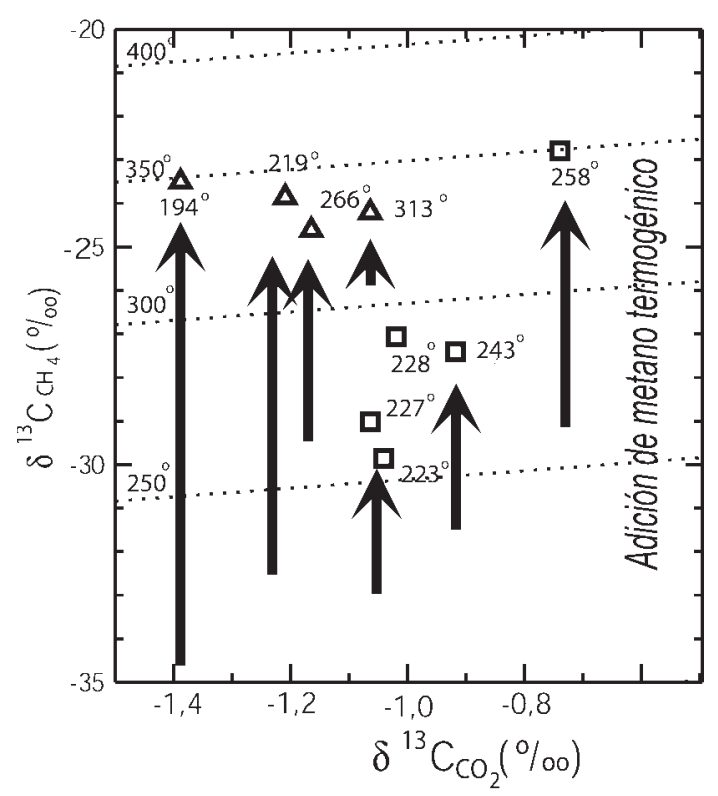

Fig. 7: Las aguas de Miravalles (triángulos) y Momotombo (cuadrados) tienen un desequilibrio entre la $\delta^{13} \mathrm{C}$ del $\mathrm{CO}_{2}$ y el $\mathrm{CH}_{4}$, debido a la reciente combinación de $\mathrm{CO}_{2}$ magmático con $\mathrm{CH}_{4}$ derivado de reacciones metamórficas en la corteza. Temperaturas de equilibrio (líneas de puntos) de Bottinga (1968). Las flechas indican la magnitud de cambio de $\delta^{13} \mathrm{C}$ debido a la adición de metano. 
de América Central reflejan diferencias en la incorporación de arcillas marinas a lo largo de la Fosa Mesoamericana (Fischer et al., 2002). Sin embargo, la estrecha relación que existe entre el nitrógeno y el metano (Snyder et al., 2002), sugiere que ambos gases son derivados de materia orgánica en la corteza, y no por la subducción (Fig. 8). No obstante, la devolatilización de material sedimentario en América Central tiene poca influencia en los valores de ${ }^{3} \mathrm{He} /{ }^{4} \mathrm{He}$, mientras que da origen al exceso de nitrógeno. Aparentemente, el flujo de helio magmático es tan alto en la región que se oculta el helio de origen sedimentario.

La distribución del nitrógeno en exceso (se resta el nitrógeno meteórico del nitrógeno total) no parece tener relación directa con el estado térmico de los campos. Por ejemplo, en Miravalles, el exceso parece estar confinado en la parte sureña del área de estudio (Fig. 9). La causa probable de esta diferencia puede ser la diferencia en composición de los sedimentos debajo del campo geotérmico que también producen cantidades menores de metano.

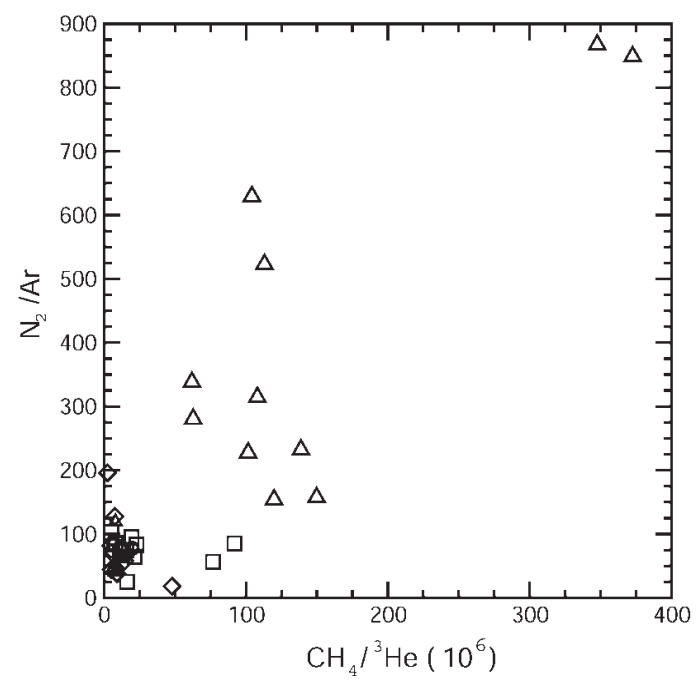

Fig. 8: Comparación de metano y nitrógeno molecular en Momotombo (triángulos). Generalmente los valores de $\mathrm{N}_{2} /$ Ar varían entre los del aire y los del agua saturada de aire. La mayoría de los pozos de Miravalles (cuadrados), Ahuachapán (diamantes) y Berlín (círculos) se parecen a las aguas termales (triángulos sólidos), que carecen de nitrógeno y metano derivado de materia orgánica.

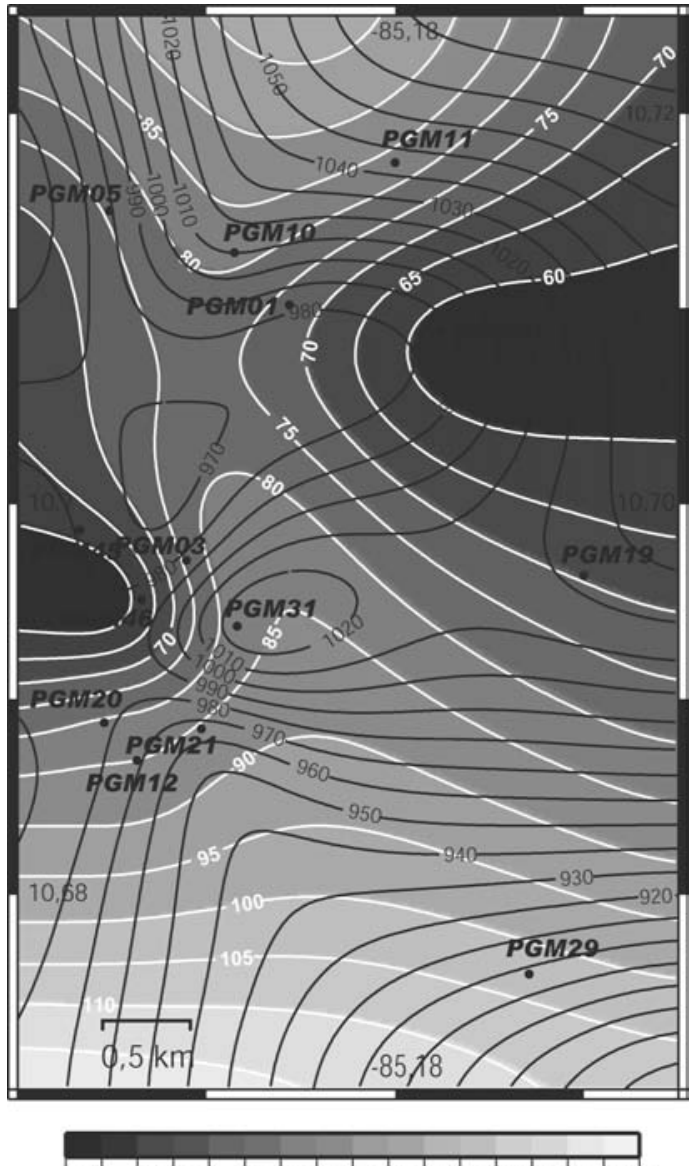

$40 \quad 45 \quad 50 \quad 55 \quad 60 \quad 65 \quad 70 \quad 75 \quad 80 \quad 85 \quad 9095 \quad 100105110115120$

$\mathrm{N}_{2} / \mathrm{Ar}$

Fig. 9: El exceso de nitrógeno se confina en el pozo más al sur de Miravalles, y es probable que se deba a contaminación de las aguas geotermales por materia orgánica.

\section{DISCUSIÓN}

La composición de los gases que emanan de los campos geotérmicos de Centroamérica se deriva de varias fuentes, que incluyen la devolatilización magmática, las aguas subterráneas y las interacciones entre las rocas en el acuífero. Dentro de los componentes magmáticos, existen los gases que se originan de la subducción de sedimentos, de los cuales más del $90 \%$ del $\mathrm{CO}_{2}$ en los gases volcánicos es de tal origen. Otros gases magmáticos, como el helio, vienen primariamente del manto y las tasas de ${ }^{3} \mathrm{He} /{ }^{4} \mathrm{He}$ indican que en la mayoría de los casos, la influencia de ${ }^{4} \mathrm{He}$ 
producida in situ en la corteza es relativamente menor. La cantidad de nitrógeno en exceso del meteórico en Centroamérica es poco usual, ya que por lo general, la regiones que tienen alto nitrógeno también tienen bajas tasas de ${ }^{3} \mathrm{He} /{ }^{4} \mathrm{He}$.

Ambos campos de producción en El Salvador, Ahuachapán y Berlín, presentan valores de $\delta^{13} \mathrm{C}$ del $\mathrm{CO}_{2}$ que son más negativos que el resto de la cordillera, y las tasas de $\mathrm{CO}_{2}{ }^{\beta} \mathrm{He}$ son más altas de lo que se podría explicar con una mezcla sencilla de gas derivado de calizas marinas con los gases del manto. Se puede explicar este fenómeno como el efecto de la pérdida previa de gases disueltos en las aguas subterráneas, lo cual aumenta el $\mathrm{CO}_{2} /{ }^{3} \mathrm{He}$. La precipitación de carbonatos dentro del campo también resulta en el fraccionamiento de ${ }^{13} \mathrm{C} /{ }^{12} \mathrm{C}$ en el bióxido de carbono, lo cual puede producir leves cambios en el $\delta^{13} \mathrm{C}$. Puesto que la explotación de Berlín es relativamente reciente, se supone que estas condiciones existieron antes de la perforación de los pozos. En Momotombo, Nicaragua, el campo se distingue por su alto contenido de metano, lo cual se asocia con el exceso de nitrógeno. La migración de la cordillera de los Marrabios hacia el oeste probablemente ha resultado en la liberación de gases asociados con la materia orgánica que yace en la depresión de Nicaragua. En el campo de Miravalles, las tasas de $\mathrm{CO}_{2} / 3 \mathrm{He}$ son elevadas, y los valores del $\delta^{13} \mathrm{C}$ se aproximan a los de las calizas, lo cual apunta hacia la influencia de la subducción de los carbonatos en la región.

Las investigaciones de la distribución de los gases dentro de los campos geotérmicos satisfacen no solamente el interés científico sobre su origen, sino que también tiene aplicaciones relevantes para el desarrollo geotérmico. El estudio de tales gases da cierta indicación de las zonas de alta influencia magmática y de los procesos relacionados con la interacción de estos con las aguas subterráneas. Futuros estudios de los cambios temporales del comportamiento de los gases dentro del campo serían importantes para indicar los efectos de la extracción y reinyección en el régimen hidrológico y termal de los campos en el curso de su explotación.

\section{AGRADECIMIENTOS}

Esta investigación no hubiera sido posible sin el apoyo logístico de las instituciones centroamericanas responsables del desarrollo geotérmico en el istmo. Agradecemos particularmente a Guillermo Alvarado y Eddy Sánchez del Instituto Costarricense de Electricidad (ICE), Karla Miranda y Ernesto Martínez de la Empresa Nicaragüense de Electricidad y José Tenorio Mejía de Geotérmica Salvadoreña (GESAL), por ayudar con la organización de los muestreos y por exponer las perspectivas en cuanto al desarrollo geotérmico en Centroamérica. Además, agradecemos a Carlos Pullinger, Mauricio Mora, Julio Álvarez y Óscar Pérez, quienes también dieron apoyo con los muestreos. Esta investigación fue financiada por la $\mathrm{Na}$ tional Science Foundation (NSF-EAR9902919).

\section{REFERENCIAS}

ADAMS, R.H., DYKSTRA, H. \& SALINAS, O., 1981: Development and reservoir analysis of Momotombo geothermal project, Nicaragua. -En: Energy resources of the Pacific Region. - AAPG Studies in Geology, 12: 27-32.

BARGAR, K.E. \& FOURNIER, R.O., 1988: Fluid-inclusion evidence for previous higher temperatures in the Miravalles geothermal field, Costa Rica. - Geothermics, 17: 681-693.

BUNDSCHUH, J., ALVARADO, G.E., RODRÍGUEZ, J.A., ROLDÁN, A.R., PALMA, J.C., ZÚÑIGA, A., REYES, E., CASTILlO, G. \& SALGADO, R.M., 2002: Resources and policy of geothermal energy in Central America. - En: CHANDRASEKHARAM, D. \& BUNDSCHUH, J. (eds.): Geothermal energy resources for developing countries. - Balkema, Lisse: 313-362.

CUÉLLAR, G., 1981: Geothermal development in El Salvador. - En: Energy Resources of the Pacific Region. AAPG Studies in Geology, 12: 21-25.

D’AMORE, F. \& MEJÍA, J.T., 1999: Chemical and physical reservoir parameters at initial conditions in Berlin geothermal field, El Salvador: A first assessment. Geothermics, 28: 45-73.

DARCE, M., BACA, D., DUARTE, M., BARBOZA, G. \& FERNÁNDEZ, A., 2000: New concepts point toward 
oil, gas potential in Nicaragua. - Oil \& Gas J. 7 Feb.: 69-73.

FARLEY, K.A. \& POREDA, R.J., 1993: Mantle neon and atmospheric contamination. - Earth Planet. Sci. Lett. 114: 325-339.

FISCHER, T.P., HILTON, D.R., ZIMMER, M.M., SHAW, A.M., SHARP, Z. \& WALKER, J.A., 2002: Subduction and recycling of nitrogen along the Central American margin. - Science, 297: 1154-1157.

FOURNIER, R.O. \& TRUESDELL, A.H., 1973: An empirical Na-K-Ca geothermometer for natural waters. - Geochim. Cosmochim. Acta, 37: 1255-1275.

GIGGENBACH, W.F., 1997: Relative importance of thermodynamic and kinetic processes in governing the chemical and isotopic composition of carbon gases in high-heatflow sedimentary basins. - Geochim. Cosmochim. Acta, 61: 3763-3785.

GIGGENBACH, W.F. \& POREDA, R.J., 1993: Helium isotopic and chemical composition of gases from volcanic-hydrothermal systems in the Philippines. Geothermics, 22: 369-380.

GIGGENBACH, W.F. \& CORRALES, R.C., 1992: Isotopic and chemical composition of water and steam discharges from volcanic-magmatic-hydrothermal systems of the Guanacaste Geothermal Province, Costa Rica. - App. Geochem. 7: 309-332.

GOFF, F.E., TRUESDELL, A.H., GRIGSBY, C.O., JANIK, C.J., SHEVENELL, L.A., PAREDES, J.R., GUTIÉRREZ, J.W., TRUJILLO, J.R., \& COUNCE, D.A., 1987: Hydrogeochemical investigation of six geothermal sites in Honduras, Central America. - 170 págs. Los Alamos Nat. Lab. Pub. LA-10785-MS, Los Alamos.

GRIGSBY, C.O., GOFF, F., TRUJILlO, P.E., COUNCE, D.A., DENNIS, B., KOLAR, J. \& CORRALES, R., 1989: Results of investigation at the Miravalles Geothermal Field, Costa Rica, Part 2: Downhole fluid sampling. - 45 págs. Los Alamos Nat. Lab Pub. LA-11510-MS, Los Alamos.

HILTON, D.R., FISCHER, T.P. \& MARTY, B., 2002: Noble gases and volatile recycling at subduction zones. - En: PORCELLI, D., BALLENTINE, C.J. \& WEILER, R. (eds.): Noble gases in cosmochemistry and geochemistry. - Rev. Min. Geochem. 47: 319-370.

HORITA, J., 2001: Carbon isotope exchange in the system $\mathrm{CO}_{2}-\mathrm{CH}_{4}$ at elevated temperatures. - Geochim. Cosmochim. Acta, 65: 1907-1919
KENNEDY, B.M., HIYAGON, H. \& REYNOLDS, J.H., 1991: Noble gases from Honduras geothermal sites. - J. Volcanol. Geotherm. Res. 45: 29-39.

KERRICK, D.M. \& CONNOLLY, J.A.D., 2000 Metamorphic devolatilization of subducted carbonate lithologies: implications for fluid fluxes and volatile recycling - [res.], AGU, EOS Trans., F1371.

LEEMAN, W.P. \& CARR, M.J., 1995: Geochemical constraints on subduction processes in the Central American Volcanic Arc: Implications of Boron Geochemistry. - En: MANN, P. (ed.): Geologic and tectonic development of the Caribbean Plate boundary in Southern Central America. - Geol. Soc. Amer. Spec. Paper, 295: 57-73.

MAINIERI, A., 2000: Costa Rica country update. - Proc. World Geothermal Congress, Kyushu-Tohoku: 313-318.

MATUS, A., MONTALVO, F., GUEVARA, W. \& MAGAÑA, M., 1999: Determination of recharge and evolutionary study in new wells at Berlin geothermal field using isotopes. - Proc. int. symposium on isotope techniques in water resources development and management, IAEA, Viena, 7 págs.

MONTALVO, F.E. \& AXELSSON, G., 2000: Assessment of reservoir changes during six years of production and reinjection in the Berlin geothermal field (El Salvador). - Proc. World Geothermal Congress, KyushuTohoku, R00876: 6 págs.

MOORE, J.L., OSBUN, E. \& STORM, P.V., 1981: Geology and Temperature Distribution of Momotombo Geothermal Field, Nicaragua. - En: Energy Resources of the Pacific Region.- AAPG Studies in Geology, 12:33-54.

PATINO, L.C., CARR, M.J. \& FEIGENSON, M.D., 2000: Local and regional variations in Central American arc lavas controlled by variations in subducted sediment input. - Contrib. Mineral. Petrol. 138: 265-283.

POREDA, R.J., JEFFREY, A.W.A., KAPLAN L.R. \& CRAIG, H., 1988: Magmatic helium in subductionzone natural gases. - Chem. Geol. 71: 199-210.

RICHET, P., BOTTINGA, Y., \& JAVOY, M., 1977: A review of hydrogen, carbon, nitrogen, oxygen, sulfur, and chlorine stable isotope fractionation among gaseous molecules. - Ann. Rev. Earth Planet. Sci. 5: 65-110.

SANO, Y. \& MARTY, B., 1995: Origin of carbon in fumarolic gases from island arcs. - Chem. Geol. 119: 265-274.

SANO, Y. \& WILLIAMS, S.N., 1996: Fluxes of mantle and subducted carbon along convergent plate boundaries. - Geophys. Res. Lett. 23: 2749-2752. 
SANO, Y., NAOTO, T., NISHIO, Y., FISCHER, T.P. \& WILLIAMS, S.N., 2001: Volcanic flux of nitrogen from the earth. - Chem. Geol. 171:263-271.

SCHOELL, M., 1988: Multiple origins of methane in the earth. - Chem. Geol. 78:1-10.

SNYDER, G.T. \& FEHN, U., 2002: The origin of iodine in volcanic fluids: ${ }^{129} \mathrm{I}$ results from the Central American Volcanic Arc. - Geochim. Cosmochim. Acta, 66: 3837-3838.

SNYDER, G., POREDA, R., HUNT, A. \& FEHN, U., 2001: Regional variations in volatile composition: isotopic evidence for carbonate recycling in the Central American volcanic arc. - Geochem. Geophys. Geosystems, 2: 2001GC000161, 25 págs.

SNYDER, G., POREDA, R., HUNT, A. \& FEHN, U., 2003: Sources of nitrogen and methane in Central American geothermal settings: Noble gas and ${ }^{129} \mathrm{I}$ evidence for crustal and magmatic volatile components. - Geochem. Geophys. Geosystems, 4: 2002GC000363, 28 págs.

STEFÁNSSON, V., 1997: Geothermal reinjection experience. - Geothermics, 26: 99-139.
VAREKAMP, J.C., KREULEN, R., POORTER, R.P.E. \& VAN BERGEN, M.J., 1992: Carbon sources in arc volcanism, with implications for the carbon cycle. Terra Nova, 4: 363-373.

VIMMERSTEDT, L., 1998: Opportunities for small geothermal projects: Rural power for Latin America, the Caribbean, and the Phillippines. - NREL,TP-21025107, 66 págs.

WELHAN, J.A., 1988: Origins of methane in hydrothermal systems. - Chem.Geol. 71: 183-198.

WELHAN, J.A. \& CRAIG, H., 1983: Methane, hydrogen and helium in hydrothermal fluids at 21 degrees $\mathrm{N}$ on the East Pacific Rise. - En: RONA, P.A., BOSTROEM, K., LAUBIER, L., SMITH, K. \& KENNETH JR., L. (eds.). Hydrothermal processes at seafloor spreading centers. Plenium, New York, 12: 391-409.

YOCK, A., 2000: Chemical and isotopic studies in the Miravalles Geothermal Field, Costa Rica. - Proc. World Geothermal Congress, Kyushu-Tohoku, 1991-1996.

ZHANG, Y. \& ZINDLER, A., 1993: Distribution and evolution of carbon and nitrogen in Earth. - Earth Planet. Sci. Lett. 117:331-345. 